\title{
Exclusive Breastfeeding Practice and Its Factors in Rural Areas of Burkina Faso
}

\author{
Léon G. Blaise Savadogo, Bernard Ilboudo, Maurice Kinda
}

Department of Epidemiology and Public Health—Child and Mother Health, Nutrition and Survival Unit—Institut Supérieur des Sciences de la Santé, Bobo Dioulasso, Burkina Faso

Email: gueswende@hotmail.com

How to cite this paper: Savadogo, L.G.B., Ilboudo, B. and Kinda, M. (2018) Exclusive Breastfeeding Practice and Its Factors in Rural Areas of Burkina Faso. Open Journal of Epidemiology, 8, 67-75.

https://doi.org/10.4236/ojepi.2018.82006

Received: December 20, 2017

Accepted: May 13, 2018

Published: May 17, 2018

Copyright $\odot 2018$ by authors and Scientific Research Publishing Inc. This work is licensed under the Creative Commons Attribution International License (CC BY 4.0).

http://creativecommons.org/licenses/by/4.0/

\begin{abstract}
Background: The recommendation of the WHO and UNICEF is that all infants should be exclusively breastfed up to 6 months of age. Identifying associated factors to exclusively breastfeeding is crucial to improve this key public health intervention. The objectives of the study were to investigate rates of exclusive breastfeeding and to identify potential associated factors. Methods: A Community based cross-sectional study design was conducted in three health districts. Cluster sampling was used. For the community based cross-sectional study, a total of 1832 infants was included. Exclusive breastfeeding have been investigated among the 563 under 6 month's infants. The associations between exclusive breastfeeding and independent variables were tested using the Chi-square test. Crude Odds ratios with $95 \%$ confidence intervals were used to investigate the factors independently associated with exclusively breastfeeding. Results: Proportion of infant $0-5.9$ months of age who are fed exclusively with breastmilk was $40.0 \%$. Ear of age for mother was found to be more likely to practice exclusive breastfeeding, COR [95CI\%] $=2.11[1.24-3.60]$ for 15 - 20 years of age and 2.0 [1.22 - 3.26]. Receiving advices, on exclusive breastfeeding was found to be more likely to practice exclusive breastfeeding, COR: $2.07,95 \%$ CI: [1.44 - 2.98]. Mothers who have practice early initiation to breastfeeding were found to be more likely to practice exclusive breastfeeding COR: 1.62, 95\% CI: [1.15 - 2.27]. Skilled delivery was found to be more likely to practice exclusive breastfeeding than non-skilled deliveries, COR: 1.66, 95\% CI: [1.16 - 2.35]. Low numbers of living children by mother, young children, were found to be more likely to practice exclusive breastfeeding. Conclusions: Exclusive breastfeeding continues to be low in rural areas of Burkina Faso. As Exclusive breastfeeding is associated to lower morbidity and mortality risk, improving the skills of health providers to give adequate feeding support to the mothers is critical for promoting exclusively breastfeeding.
\end{abstract}




\section{Keywords}

Prevalence, Exclusive Breastfeeding, Rural Areas, Burkina Faso

\section{Background}

The recommendation of the WHO and UNICEF is that all infants should be exclusively breastfed up to 6 months of age [1] [2]. Exclusive breastfeeding among under 6 month's infants is one of the most effective interventions to reduce child mortality [3] [4]. Exclusive breastfeeding has benefits to child health and development.

Breast milk is the best food for a child's health, growth and development during the 6 first months. All the vitamins, minerals, enzymes and antibodies need by a child are provided by the breast milk [2].

Breast milk is an unequalled way of provision of best food for infant's healthy growth and development [5] and for reduction of infant morbidity and mortality [6] [7]. Exclusive breastfeeding is benefit to the mother, preventing post-partum hemorrhage and allows spacing of pregnancies [1].

Despite all recommendations and benefits, according to the UNICEF, exclusive breastfeeding rate for the first six month has not changed significantly since 1990 and is around 36\% [8] [9].

Despite these benefits, less than $50 \%$ of infants (WHO target) in resource limited settings are exclusively breastfeed. Identifying associated factors to exclusively breastfeeding is crucial to improve this key public health intervention.

The objectives of the study were to investigate rates of exclusive breastfeeding among under 6 months and to identify potential factors associated among Burkina Faso rural settings mothers.

\section{Methods}

A Community based cross-sectional study design was conducted in three districts of East region (Manni and Gayéri) and center-north region (Boulsa) from November to December 2011. Manni and Gayéri are two of the six districts of the East region. Boulssa is one of the three district of the center-north region. All rural mothers who have children less than 24 months were considered as source population. The procedures for determining survey sample size are designed to take into account the requirements for a follow-up survey round of a program evaluation as initial round in the concerned districts.

As the survey was for implementing feeding intervention, we used the percentage of infants who were stunted as the indicator for calculating the sample size in each district.

The following basic equation has been used to calculate the required sample size for each district.

$$
n=D *\left[(Z \alpha+Z \beta)^{2} *(P 1 *(1-P 1)+P 2 *(1-P 2)) /(P 2-P 1)^{2}\right]
$$


Cluster sampling, as probability sampling methods was used. In each district, villages were the clusters. Size of each cluster was available from the last population census to sample selection. Probability-proportional-to-size (PPS) selection procedures were used.

Random-walk method for selecting sample households has been used.

The data were collected by using a structured and pretested interview questionnaire.

The study used one major questionnaire with socio-demographic factors, maternal factors, child related factors and characteristics of child feeding practices were included in the survey.

For the community based cross-sectional study, total sample size was 1832 infants.

For the investigation of exclusive breastfeeding, total sample size of under 6 month's infants was 563.

Descriptive statistics were used. The associations between exclusive breastfeeding and independent variables were tested using the Chi-square test. Crude Odds ratios (COR) with $95 \%$ confidence intervals (95\% CI) were used to investigate the factors independently associated with exclusively breastfeeding. All of the results were considered statistically significant at $\mathrm{P}<0.05$.

Ethical clearance was obtained from Burkina Faso ethical committee for health research. At the time of data collection, a verbal and written consent was taken from the participants.

\section{Results}

\subsection{Socio-Demographic Characteristics of the Participants}

Among the 563 mother-infant s, the majority (52.8\%) of mothers were in the age group 21 - 30 years. The mean age of mothers was $25.3 \pm 6.2$ years.

The highest proportion of mothers $(90.8 \%)$ had not attended primary school. All of mothers of the mothers were housewives. The average number of living children with mother was 3 .

The average number of pregnancies per woman was 3.7. More than $80 \%$ of mothers had more than one pregnancy (Table 1 ).

The mean age of infants was $2.95 \pm 1.6$ months, and $49.4 \%$ were boys and $50.6 \%$ girls.

The majority (76.6\%) of children had health care carte showing that they have contact with health facilities for care.

\subsection{Advices Received during Pregnancy}

The majority (78.2\%) of mothers received antenatal care. During pregnancy, $62.0 \%$ of mothers have received advices on exclusively breastfeeding; $52.8 \%$ received advice on child growth and $52.8 \%$ on child feeding.

Out of participants, $62.2 \%$ of mothers have been delivered on health facilities by skilled care providers. 
Table 1. Baseline characteristics of the participants.

\begin{tabular}{ccc}
\hline & $\mathbf{n}$ & $\%$ \\
\hline Mothers age (years) & & \\
\hline $15-20$ & 162 & 28.8 \\
$21-30$ & 297 & 52.8 \\
$\geq 31$ & 104 & 18.4 \\
\hline Formal school education of mothers & & \\
Yes & 52 & 9.2 \\
No & 511 & 90.8 \\
\hline 1 & & \\
\hline Living children number by mother & 146 & 25.9 \\
$2-3$ & 235 & 41.7 \\
$\geq 6$ & 154 & 27.4 \\
\hline Number of pregnancies by mother & 28 & 5.0 \\
\hline 1 & & \\
$2-6$ & 118 & 21.0 \\
$\geq 6$ & 381 & 67.7 \\
\hline & 64 & 11.4 \\
\hline
\end{tabular}

Out of participants, $43.7 \%$ of infants have been early breastfeed (Table 2).

\subsection{Exclusive Breastfeeding}

Proportion of infant 0 - 5.9 months of age who are fed exclusively with breastmilk was $40.0 \%$.

Table 3 gives this percentage at different age of infants.

The percentage of exclusively breastfeed infants decreases from $46.9 \%$ at 0 1.9 months of age to $29.0 \%$ at $4-5.9$ months of age (Figure 1 ).

The percentage of exclusively breastfeed infants decreases from 1 month to 6 months of age (Figure 2).

\subsection{Predominant Breastfeeding}

Proportion of infants 0 - 5.9 months of age who are predominantly breastfed (infants who received breastmilk as predominant source of nourishment during previous day, but also receive other fluid) was $83.5 \%$ (470/563).

\subsection{Exclusive Breastfeeding Factors}

Table 4 shows the results of bivariate analyses.

Age of mothers, advices on exclusive breastfeeding during pregnancy, early breastfeeding initiation, delivery by skilled care providers, number of living children by mother and children age were associated with exclusive breastfeeding.

Having less than 31 year of age for mother was found to be more likely to practice exclusive breastfeeding, COR: 2.11, 95\% CI: [1.24 - 3.60] for 15 - 20 years of age and COR: 2.0, 95\% CI: [1.22 - 3.26] for $21-30$ years, $p=0.01$. 
Table 2. Care and advices during the pregnancy period.

\begin{tabular}{ccc}
\hline & $\mathbf{n}$ & $\%$ \\
\hline Number of antennal care contact & & \\
\hline 0 & 123 & 21.8 \\
$1-2$ & 75 & 13.3 \\
$3-4$ & 365 & 64.8 \\
\hline Advice on Exclusively breastfeeding & & \\
\hline Yes & 349 & 62.0 \\
No & 214 & 38.0 \\
\hline Advice on child growth & & \\
\hline Nos & 297 & 52.8 \\
\hline Advice on child feeding & 266 & 47.2 \\
\hline Yes & & \\
No & 297 & 52.8 \\
\hline Skilled birth & 266 & 47.2 \\
\hline Yes & & \\
No & 350 & 62.2 \\
\hline Yes & 213 & 37.8 \\
\hline No & & \\
\hline
\end{tabular}

Table 3. Exclusive breastfeeding indicators.

\begin{tabular}{lcc}
\hline & n & $\%$ \\
\hline Proportion of infant 0 - 1.9 months of age who are fed exclusively with breastmilk & 213 & 46.9 \\
Proportion of infant 2 - 3.9 months of age who are fed exclusively with breastmilk & 188 & 41.5 \\
Proportion of infant 4 - 5.9 months of age who are fed exclusively with breastmilk & 162 & 29.0 \\
Proportion of infant 0 - 3.9 months of age who are fed exclusively with breastmilk & 401 & 44.4 \\
Proportion of infant 0 - 5.9 months of age who are fed exclusively with breastmilk & 563 & 40.0 \\
\hline
\end{tabular}

Receiving advices, during pregnancy, on exclusive breastfeeding was found to be more likely to practice exclusive breastfeeding, COR: $2.07,95 \%$ CI: [1.44 2.98], $\mathrm{p} \leq 0.001$.

Mother who have practice early initiation to breastfeeding were found to be more likely to practice exclusive breastfeeding COR: 1.62, 95\% CI: [1.15 - 2.27], $\mathrm{p}=0.004$.

Skilled delivery was found to be more likely to practice exclusive breastfeeding than non-skilled deliveries, COR: 1.66, 95CI\%: [1.1 6 - 2.35], $\mathrm{p}=0.004$.

Low number of living children by mother, young children, was found to be more likely to practice exclusive breastfeeding.

\section{Discussion}

The WHO sets optimal breastfeeding prevalence at $90 \%$. In the low-income countries, only $39 \%$ of children aged less than 6 months are exclusively breastfed 


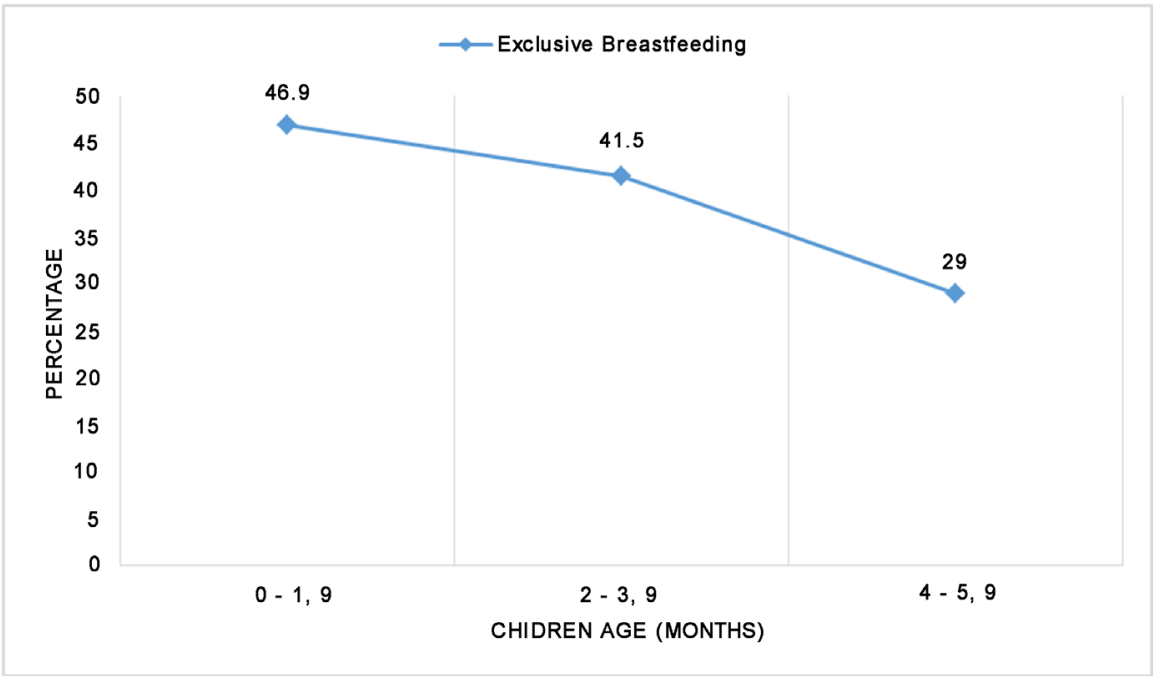

Figure 1. Percentage of exclusive breastfeeding at different periods of child life.

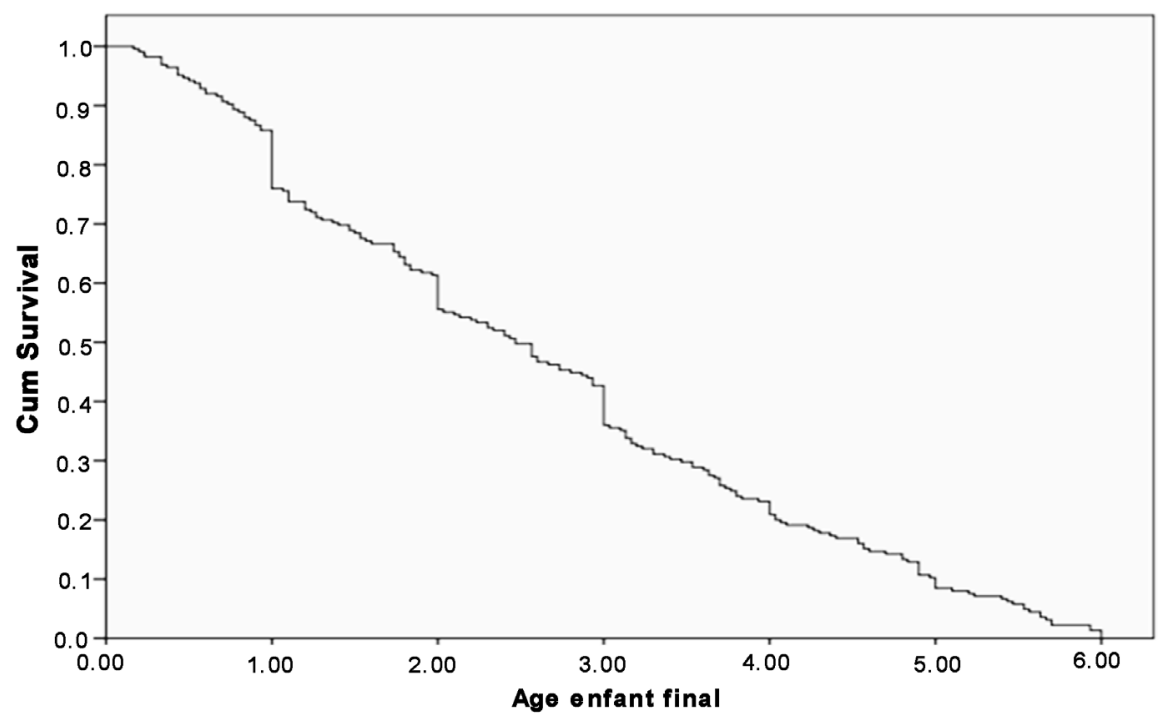

Figure 2. Decrease of the percentage of exclusive breastfeeding during the six first months.

[1]. The prevalence of exclusively breastfeeding was $40.0 \%$ in this study. The prevalence of exclusively breastfeeding [10] for West Africa, East Africa, Central Africa and Southern Africa was 32.64\% (95\% CI 25.85 to 39.43), 53.48\% (95\% CI 40.14 to 66.82 ), $23.70 \%$ (95\% CI 5.37 to 42.03 ) and $56.57 \%$ (95\% CI 53.20 to 59.95), respectively. In West Africa, Cote d'Ivoire reported the lowest prevalence of exclusively breastfeeding of $13.15 \%$. The corresponding prevalence reported in East Africa, Central Africa and Southern Africa was 13.45\% (Comoros), 6.04\% (Gabon) and 48.66\% (Namibia).

In this study, the prevalence of predominant breastfeeding was high (83.5\%). The prevalence of predominant breastfeeding [10] for West Africa, East Africa, Central Africa and Southern Africa was $43.67 \%$ (95\% CI 37.22 to 55.52 ), $17.63 \%$ (95\% CI 12.70 to 22.55 ), $31.63 \%$ (95\% CI 19.77 to 43.48 ) and $13.07 \%$ (95\% CI 
Table 4. Univariate analysis showing association of factors with exclusive breastfeeding.

\begin{tabular}{|c|c|c|c|c|}
\hline & $\mathbf{n}$ & & OR $[95 \% \mathrm{CI}]$ & p-value \\
\hline \multicolumn{5}{|c|}{ Mothers age (years) } \\
\hline $15-20$ & 162 & 43.8 & $2.11[1.24-3.60]$ & \multirow{3}{*}{0.01} \\
\hline $21-30$ & 297 & 42.4 & $2.0[1.22-3.26]$ & \\
\hline$\geq 31$ & 104 & 26.9 & 1.0 & \\
\hline \multicolumn{5}{|c|}{ Formal school education of mothers } \\
\hline Yes & 52 & 42.3 & $1.11[0.62-1.98]$ & \multirow{2}{*}{0.71} \\
\hline No & 511 & 39.7 & 1.0 & \\
\hline \multicolumn{5}{|c|}{ Children sex } \\
\hline Girls & 278 & 40.6 & $1.05[0.75-1.48]$ & \multirow{2}{*}{0.74} \\
\hline Boys & 285 & 39.3 & 1.0 & \\
\hline \multicolumn{5}{|c|}{ Advice on Exclusively breastfeeding } \\
\hline Yes & 349 & 46.4 & $2.07[1.44-2.98]$ & \multirow{2}{*}{$\leq 0.001$} \\
\hline No & 214 & 29.4 & 1.0 & \\
\hline \multicolumn{5}{|c|}{ Skilled birth } \\
\hline Yes & 350 & 47.5 & $1.66[1.16-2.35]$ & \multirow{2}{*}{0.004} \\
\hline No & 213 & 35.2 & 1.0 & \\
\hline \multicolumn{5}{|c|}{ Early breastfeeding initiation } \\
\hline Yes & 246 & 48.2 & $1.62[1.15-2.27]$ & \multirow{2}{*}{0.004} \\
\hline No & 317 & 36.6 & 1.0 & \\
\hline \multicolumn{5}{|c|}{ Number of pregnancies by mother } \\
\hline 1 & 118 & 40.7 & $1.89[0.97-3.68]$ & \multirow{3}{*}{0.06} \\
\hline $2-5$ & 381 & 42.0 & $2.00[1.10-3.61]$ & \\
\hline$\geq 6$ & 64 & 26.6 & 1.0 & \\
\hline \multicolumn{5}{|c|}{ Number of living children } \\
\hline 1 & 146 & 40.4 & $8.81[2.01-38.56]$ & \multirow{4}{*}{0.02} \\
\hline $2-3$ & 235 & 44.3 & $10.32[2.39-44.48]$ & \\
\hline $4-5$ & 154 & 39.0 & $8.29[1.9-36.24]$ & \\
\hline$\geq 6$ & 28 & 7.1 & 1.0 & \\
\hline \multicolumn{5}{|c|}{ Children Age (months) } \\
\hline $0-1.9$ & 213 & 46.9 & $1.60[1.02-2.51]$ & \multirow{3}{*}{0.002} \\
\hline $2-3.9$ & 188 & 41.5 & $2.16[1.4-3.33]$ & \\
\hline $4-5.9$ & 162 & 29.0 & 1.0 & \\
\hline
\end{tabular}

10.77 to 15.38), respectively. Countries with the lowest prevalence of PBF were Gambia (21.75\%) in West Africa, Kenya (6.52\%) in East Africa, Gabon (19.62\%) in Central Africa and Lesotho (8.25\%) in Southern Africa.

The target of WHO for exclusively breastfeeding is more than 50\% [11]. The overall exclusively breastfeeding prevalence across the countries was less than $50 \%$.

There is evidence that infants who are exclusively breastfeed during the first months have lower morbidity and mortality risk, due to reductions in deaths caused by infectious diseases [6] [7] [12] [13] [14].

Despite these benefits, our finding show that the prevalence of exclusively breasfeeding was lower than the WHO target of 50\%. It is similar than recorded prevalence of many low incomes countries [10].

Young mothers aged less than 31 year; receiving advice on exclusive breast- 
feeding; mothers who have practice early initiation to breastfeeding; skilled delivery; and mother with low number of living children; were found to be more likely to practice exclusive breastfeeding. Utilization of health facility services, support from the health personnel, and counseling on breastfeeding support can increase the practice of exclusive breastfeeding [15] [16].

Our study found that the overall/pooled prevalence of predominant breastfeeding is high $(83.3 \%)$. Target is a lower prevalence, as predominant breastfeeding has a risk effect on an infant's health. Optimal breastfeeding prevalence is at $90 \%$ with less than $10 \%$ of predominant breastfeeding.

\section{Conclusion}

This study demonstrates that exclusive breastfeeding continues to be low in rural areas of Burkina Faso, and less than target level of 50\%. But the prevalence of predominant breastfeeding was high (83.3\%). Age of mothers, advices on exclusive breastfeeding during pregnancy, early breastfeeding initiation, delivery by skilled care providers, number of living children by mother and children age were associated with exclusive breastfeeding practice.

As exclusive breastfeeding is associated to lower morbidity and mortality risk, improving the skills of health providers to give adequate feeding support to the mothers during prenatal care visits and after delivery is critical for promoting exclusively breastfeeding.

\section{Acknowledgements}

The authors are grateful to the mothers and their families for participation in the study. Thanks are also due to the staff of the three health Districts

\section{Author Contributions}

All authors contributed to study design, literature review, data collection, data analysis and interpretation, and writing. All authors revised and agreed on the views expressed in the manuscript.

\section{Conflicts of Interest}

The authors declare no conflict of interest.

\section{References}

[1] UNICEF. Infant and Young Child Feeding. UNICEF New York 2011. https://www.unicef.org/nutrition/files/Final_IYCF_programming_guide_2011.pdf

[2] USAID (2012) Nutrition in the First 1000 Days. USAID, New York.

[3] Jones, G., Steketee, R.W., Black, R.E., Bhutta, Z.A. and Morris, S.S., Bellagio Child Survival Study G (2003) How Many Child Deaths Can We Prevent This Year? Lancet, 362, 65-71.

[4] Victora, C.G., Bahl, R., Barros, A.J., Franca, G.V., Horton, S., Krasevec, J., et al. (2016) Breastfeeding in the 21st Century: Epidemiology, Mechanisms, and Lifelong Effect. Lancet, 387, 475-490. https://doi.org/10.1016/S0140-6736(15)01024-7 
[5] WHO. Exclusive Breastfeeding. Nutrition. http://www.who.int/nutrition/topics/exclusive_breastfeeding/en/

[6] León-Cava, N., Lutter, C., Ross, J., et al. (2002) Quantifying the Benefits of Breastfeeding: A Summary of the Evidence. PAHO, Washington DC.

[7] Horta, B.L., Bahl, R., Martines, J.C., et al. (2007) Evidence on the Long-Term Effects of Breastfeeding: Systematic Reviews and Meta-Analyses. World Health Organization, Geneva.

[8] Ip, S., Chung, M., Raman, G., Chew, P., Magula, N., DeVine, D., et al. (2007) Breastfeeding and Maternal and Infant Health Outcomes in Developed Countries. Evidence Report/Technology Assessment, 153, 1-186.

[9] Yalcin, S.S., Yalcin, S. and Kurtulus Yigit, E. (2014) Determinants of Continued Breastfeeding beyond 12 Months in Turkey: Secondary Data Analysis of the Demographic and Health Survey. The Turkish Journal of Pediatrics, 56, 581-91.

[10] Ellis, J. (2005) Neonatal Hypothermia. Journal of Neonatal Nursing, 11, 76-82. https://doi.org/10.1016/j.jnn.2005.04.010

[11] WHO. Global Nutrition Targets 2025. http://www.who.int/nutrition/global-target-2025/ en/

[12] Black, R.E., Allen, L.H., Bhutta, Z.A., et al. (2008) Maternal and Child Undernutrition: Global and Regional Exposures and Health Consequences. Lancet, 371, 243-260. https://doi.org/10.1016/S0140-6736(07)61690-0

[13] Edmond, K.M., Zandoh, C., Quigley, M.A., et al. (2006) Delayed Breastfeeding Initiation Increases risk of Neonatal Mortality. Pediatrics, 117, e380-e386. https://doi.org/10.1542/peds.2005-1496

[14] Mullany, L.C., Katz, J., Li, Y.M., et al. (2008) Breast-Feeding Patterns, Time to Initiation, and Mortality Risk among Newborns in Southern Nepal. The Journal of Nutrition, 138, 599-603. https://doi.org/10.1093/jn/138.3.599

[15] Yotebieng, M., Chalachala, J.L., Labbok, M. and Behets, F. (2013) Infant Feeding Practices and Determinants of Poor Breastfeeding Behavior in Kinshasa, Democratic Republic of Congo: A Descriptive Study. International Breastfeeding Journal, 8, 11. https://doi.org/10.1186/1746-4358-8-11

[16] Yotebieng, M., Labbok, M., Soeters, H.M., Chalachala, J.L., Lapika, B., Vitta, B.S. and Behets, F. (2015) Ten Steps to Successful Breastfeeding Programme to Promote Early Initiation and Exclusive Breastfeeding in DR Congo: A Cluster-Randomised Controlled Trial. The Lancet Global Health, 3, e546-e555. https://doi.org/10.1016/S2214-109X(15)00012-1 